\title{
Volatile inhibitors of atmospheric corrosion of ferrous and nonferrous metals III. VNKh-L-408 inhibitor: basics of production and application technology
}

\author{
A.I. Altsybeeva, V.V. Burlov, N.S. Fedorova and T.M. Kuzinova \\ All-Russia Research Institute of Petrochemical Processes, ul. Pulkovskaya 10, \\ St. Petersburg, 192148 Russian Federation \\ E-mail: altsybeeva@yandex.ru
}

\begin{abstract}
Criteria for selection of inhibitors based on their metal corrosion inhibition factors in an environmental chamber have been proposed. It has been demonstrated that a wide range of ferrous and nonferrous metals are efficiently protected by 1-(morpholino)(phenyl)methylbenzotriazole conventionally named as VNKh-L-408. The basics of its production and application technology have been developed.
\end{abstract}

Key words: criteria for selection of inhibitors, production and application technology, VNKh-L-408 inhibitor.

Received: December 5, 2012

doi: 10.17675/2305-6894-2013-2-1-009-016

In [1], we have demonstrated that thermally stable molecular compounds such as Schiff and Mannich bases are promising as volatile inhibitors of atmospheric corrosion (VIACs) of ferrous and nonferrous metals. When choosing an inhibitor that can be recommended for development of the commercial production technology, we considered the range of metals protected by the inhibitor and fast protection for dissimilar metals achievable by such inhibitor.

Accelerated corrosion tests are carried out under the assumption that the atmospheric corrosion of metals follows the same mechanism, whether under natural conditions or in an environmental chamber. In the latter case, several cycles are usually required to initiate corrosion. The degree of corrosion depends on the resistance of the metal to atmospheric corrosion, provided that the test duration is the same.

According to GOST (State Standard) 9.509 [2], it is nearly always required to choose a proper test cycle (for a specified testing method) from test results for some protective agent with a known protection period certified by industrial testing. The proper test cycle in an environmental chamber was determined from the protection periods of the NDA and KhTsA inhibitors specified in GOST 9.014 [3]. One test cycle corresponds to $\sim 3.5-4$ months of storage in an unheated warehouse or under a shed in an industrial coastal town.

Because dissimilar metals differently corrode in air in the absence of inhibitors, it is advisable to assess the efficiency of an inhibitor from the inhibition factors of metal 
corrosion testing for 15 cycles in an environmental chamber (about five years under natural conditions). In our opinion, an efficient versatile VIAC should have the following inhibition factors $\gamma: \geq 25$ for steel, $\geq 50$ for copper, $\geq 200$ for brass, and $\geq 75$ for bronze, and complete suppression of corrosion of D-16 aluminum and MA-8 magnesium. Since versatile inhibitors should also at least not initiate the corrosion of cadmium- and zincplated surfaces, a level of $\gamma \geq 1.5$ is proposed for zinc and cadmium.

1-(Morpholino)(phenyl)methylbenzotriazole (VNKh-L-408 inhibitor) is one of the most efficient VIACs, despite its low volatility (calculated $P^{0}=0.23 \cdot 10^{-7} \mathrm{mmHg}$ at $20^{\circ} \mathrm{C}$ ). Corrosion tests showed that VNKh-L-408 is efficient against the atmospheric corrosion of various steels and cast irons; oxidized, chromium-plated, and nickel-plated surfaces of steel products; copper and its alloys (various brasses and bronzes); cadmium (including cadmium-plated surfaces); zinc (including zinc-plated surfaces); aluminum alloys, magnesium alloys, and $\mathrm{Al}-\mathrm{Mg}$ alloys; lead and its alloys; silver and silver-plated items. This inhibitor is highly fungistatic (i.e., it suppresses the rise and growth of the most commonly encountered fungi on metal items).

The inhibition factors of VNKh-L-408 for all the metals tested vastly exceed the proposed criteria (see Table 4 [1]), which made it expedient to develop the technology of production and application of this inhibitor.

The VNKh-L-408 inhibitor is produced by complex condensation of morpholine, benzotriazole, and benzaldehyde. This Mannich-type reaction involves morpholine as a secondary amine, benzotriazole as a compound with an acidic hydrogen atom, and benzaldehyde as an aldehyde component. The rate of this exothermic reaction is very high and is limited by the feed rate of the reagents. The VNKh-L-408 inhibitor has been patented [4].

Reactions involving heterocyclic compounds (e.g., pyrrole and its derivatives, benzimidazole, benzotriazole, etc.), secondary amines, and formaldehyde have been described in [5]. Here we employed the method proposed by Bachman [5], with benzaldehyde in place of formaldehyde. However, the yield of the target product was not very high, so we modified the method by changing the reaction conditions and the sequence of addition of the reagents.

The technology for the synthesis of VNKh-L-408 was developed under laboratory conditions and then tried out at the pilot production plant of the All-Russia Research Institute of Petrochemical Processes. A flask equipped with a stirrer, a reflux condenser, and a dropping funnel was charged with a solution of benzotriazole in isopropyl alcohol. Then benzaldehyde and morpholine were successively added with stirring at $50^{\circ} \mathrm{C}$. The reaction mixture was kept at $55-65^{\circ} \mathrm{C}$ for $3 \mathrm{~h}$ and cooled to $0-5^{\circ} \mathrm{C}$ for one hour. The white precipitate that formed was filtered off on a Büchner funnel, washed with isopropyl alcohol, and dried in air. The mother liquor was properly treated to recover isopropyl alcohol for its reuse. 
The structural formula of the VNKh-L-408 inhibitor

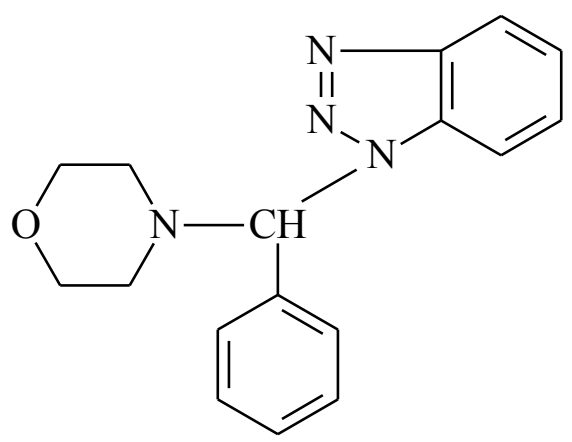

was confirmed by IR spectroscopy and elementary analysis.

The IR spectrum of benzotriazole shows medium-intensity absorption bands at 30593024 and $1580 \mathrm{~cm}^{-1}\left(\mathrm{NH}_{\text {pyrrole }}\right)$ and intense bands at $1647-1642 \mathrm{~cm}^{-1}$ (on average, $\left.1645 \mathrm{~cm}^{-1}\right)(\mathrm{N}=\mathrm{N}[6,7])$.

The shift of the band of the N=N group in the IR spectrum of VNKh-L-408 to 1604$1584 \mathrm{~cm}^{-1}$ (on average, $1594 \mathrm{~cm}^{-1}$ ) is due both to conjugation with the benzene ring and the effect of the lone electron pair of the $\mathrm{N}$ atom of the morpholine ring.

The positions of the characteristic bands in the IR spectrum of morpholine: 2955$2930 \mathrm{~cm}^{-1}$ (on average, $2942 \mathrm{~cm}^{-1}$ ) (three oxygen-related bands); $840-837 \mathrm{~cm}^{-1}$ (two oxygen-related bands); $1140-1095 \mathrm{~cm}^{-1}$ (on average, $1120 \mathrm{~cm}^{-1}$ ) (four bands due to the large rings containing epoxy groups); $2830-2820 \mathrm{~cm}^{-1}$ (on average, $2825 \mathrm{~cm}^{-1}$ ) (two bands due to the morpholine $\mathrm{N}$ atom) in the IR spectrum of the inhibitor remain nearly unchanged (2954, 851, 1111, and $2826 \mathrm{~cm}^{-1}$, respectively).

Corrosion tests revealed that optimum protection is achieved if the content of the active component in the inhibitor is at least $85 \mathrm{wt} . \%$ and the morpholine content is $4 \mathrm{wt} . \%$ maximum. Admixtures of benzotriazole and benzaldehyde have no adverse effect on the protective properties of the final product. However, if the content of free benzaldehyde exceeds $5 \mathrm{wt} . \%$, nonferrous metals can darken because of oxidation of benzaldehyde into corrosive benzoic acid.

While developing the pilot scale production technology of VNKh-L-408, we studied the effects of the temperature, reaction time, ratio of the components, solvent, and possibility of its recycling without recovery or washing on the yield, content of the active component in the final product, and its protective properties.

Acetone, ethanol, isopropyl alcohol, and hexane were tried as solvents. We found that the choice of the solvent depends on the solubility of benzotriazole because homogenization is required to complete the reaction. However, both ethanol and acetone dissolve the inhibitor very well, so its yield does not exceed $40 \%$. The solubility of benzotriazole in hexane is insufficient and the final product contains a considerable admixture of the solvent. Moreover, the product obtained in hexane has poor protective properties. Isopropyl alcohol proved to be the optimum solvent for the synthesis of VNKhL-408. The other conditions for its pilot scale production are as follows: the morpholine : 
benzaldehyde : benzotriazole molar ratio is $1: 1.5: 1$, the reaction time is $3.5 \mathrm{~h}$, the temperature is $50-60^{\circ} \mathrm{C}$, isopropanol is used as the solvent.

The yield of the inhibitor with respect to morpholine is $\sim 85 \%$. The used solvent is recovered by removal of the azeotropic mixture with water in a column at atmospheric pressure, so isopropyl alcohol is recycled.

The pilot scale synthesis of the VNKh-L-408 inhibitor is a batch process consisting of the following steps:

1. Synthesis of the inhibitor

2. Isolation of the inhibitor from the mother liquor

3. Drying of the product

4. Recovery of the solvent.

Table 1 presents data on the consumption of the starting reagents per ton of the VNKh-L-408 inhibitor. To draw up the material balance, we used the following data:

- conversion of morpholine is $85 \%$;

- conversion of benzotriazole is $75 \%$;

- conversion of benzaldehyde is $62 \%$;

- yield of the VNKh-L-408 inhibitor is $80-85 \%$.

Table 1. Consumption of the starting materials in the production of one ton of the VNKh-L-408 inhibitor.

\begin{tabular}{lcc}
\hline Starting material & Unit of measure & Amount per ton of the inhibitor \\
\hline Morpholine & ton & 0.373 \\
Benzaldehyde & ton & 0.639 \\
Benzotriazole & ton & 0.511 \\
Isopropyl alcohol & ton & 0.423 \\
\hline
\end{tabular}

The laboratory results were verified at the pilot production plant of the All-Russia Research Institute of Petrochemical Processes. The data obtained were used to develop technological regulations for the pilot scale production of the inhibitor.

The inhibitor application technology developed by us is based on the approaches specified in GOST 9.014 [3] for protection by volatile inhibitors, with allowance for worldwide and domestic experience in preserving and protecting metal items [8] as well as for the physicochemical and protective properties of the inhibitor.

The main ways to use the VNKh-L-408 inhibitor for the protection of metal items from atmospheric corrosion are:

- in the form of powder inside sachets placed into the cavities of metal items and devices and in other enclosures to be protected from corrosion;

- in the form of powder electrostatically applied to the surface of a metal item or into the inside of an enclosure; 
- in the form of "lingal" (inhibitor applied on alumina granules up to $10-12 \mathrm{wt} . \%$ of the resulting material);

- in the form of "tablin" (pellets of compressed inhibitor, up to 95-98 wt.\%);

- in the form of "granlin" (shaped granules containing the inhibitor (at least $95 \mathrm{wt} . \%$ ) and a binder).

The results of the corrosion tests of steel St3 and copper M1 in the presence of the VNKh-L-408 inhibitor in all its recommended forms are summarized in Table 2 (see also Table 4 in [1]). It follows from the data obtained that:

- electrostatic application of the inhibitor to the surface (or into the inside) of an object to be protected provides the best protection. When the concentration of the inhibitor is $\sim 10 \mathrm{~g} / \mathrm{m} 2$ of the surface (which corresponds to $\sim 60 \mathrm{~g} / \mathrm{m}^{3}$ of air), its protective value is higher than that achieved in the presence of its shaped forms ("granlin", "lingal", and "tablin") and is the same as for its powder in sachets suspended inside a metal object (this is one of the most laborious methods);

- an increase in the inhibitor concentration in an enclosure to be protected increases the inhibition factors for steel and copper corrosion; however, when used in a shaped form, this often lowers the inhibition factor for the corrosion of copper alloys;

- electrostatic application of the inhibitor, as well as its use in "granlin" or "lingal" forms, makes it more versatile and increases its protective value for such metals as zinc, cadmium, aluminum, and its alloys with magnesium compared to powdered VNKh-LF-408, the concentration in the air being nearly equal;

- the norms regulating the use of an inhibitor and the choice of its appropriate form depend on the list of metals to be protected and on the desired preservation period of a metal item prior to its use.

Table 2. Inhibition factors obtained for the corrosion of St3 steel and M1 copper in an environmental chamber in the presence of various forms of VNKh-L-408. The concentration of the inhibitor in air is $50 \mathrm{~g} / \mathrm{m}^{3}$. The test duration is 15 cycles.

\begin{tabular}{lcc}
\hline Form of use & St3 Steel & M1 Copper \\
\hline Electrostatically applied powder & 250 & 100 \\
Powder in sachets & 125 & 80 \\
"Granlin" & 70 & 75 \\
"Tablin" & 37 & 75 \\
"Lingal" & 27 & 70 \\
\hline
\end{tabular}

The approximate amounts of the inhibitor and inhibitor-based materials per cubic meter of an item to be protected are as follows (provided that the part or its package are hermetically sealed): 
- 50-100 g for powder, "tablin", or "granlin";

- up to $10 \mathrm{~g} / \mathrm{m} 2$ of the surface for an electrostatically applied powder;

- 100-500 g for "lingal".

As prescribed by GOST 9.014 [3] and suggested by preservation experience [8], the articles preserved with inhibitors must be kept for at least $72 \mathrm{~h}$ under conditions preventing moisture condensation. According to [9], the 72-hour passivation period is required for the VIAC vapor to saturate the enclosed space and for its concentration in the condensed moisture film on the metal surface to reach a minimum protective value ensuring metal passivation. The calculated passivation times for metal items in the presence of various forms of the VNKh-LF-408 inhibitor are given in Table 3.

Table 3. Calculated passivation times (days) for metal items kept in the presence of the inhibitor VNKhLF-408 under the conditions preventing moisture condensation [9].

\begin{tabular}{lcccccc}
\hline & $\begin{array}{c}\text { Total rated } \\
\text { Form of use }\end{array}$ & $\begin{array}{c}\text { Passivation time (days): } \\
\text { consumption } \\
\text { of the inhibitor, } \\
\mathbf{g} / \mathbf{m}^{\mathbf{3}} \text { of air }\end{array}$ & \multicolumn{5}{c}{$\begin{array}{c}\text { number of portions added up to give the total rated } \\
\text { consumption of the inhibitor: }\end{array}$} \\
& 50 & $\mathbf{1}$ & $\mathbf{2}$ & $\mathbf{3}$ & $\mathbf{4}$ & $\mathbf{5}$ \\
\hline Powder & 100 & 7.6 & 4.8 & 3.7 & 3 & 2.6 \\
Same & 100 & 9.3 & 5.8 & 4.5 & 3.7 & 3.2 \\
Lingal & 200 & 7.4 & 4.7 & 3.5 & 2.9 & 2.5 \\
Same & 50 & 11.2 & 7.1 & 5.4 & 4.45 & 3.9 \\
Tablin & 100 & 8.9 & 5.6 & 4.3 & 3.5 & 3.1 \\
Same & 150 & 7.8 & 4.9 & 3.7 & 3.1 & 2.7 \\
Same & & & & & &
\end{tabular}

By analogy with the VNKh-L-20 inhibitor industrially tested for a long period of time, one can predict that the VNKh-L-408 inhibitor will be efficient for up to ten years, provided that the metal items to be protected are hermetically sealed. This inhibitor suppresses the rise and growth of the most commonly encountered fungi, thus protecting the articles from biological damage as well.

The VNKh-L-408 inhibitor should be used in conformity with GOST 9.014 [3] (protection variant VZ-14, Appendix 8). The following basic rules for preservation of parts, articles, and machinery with volatile inhibitors should be observed:

- only dry and clean metal items should be preserved;

- the inhibitor in any form (powder, "tablin" ("granlin"), or "lingal") should be placed in an internal cavity of an item or in a packing enclosure, with subsequent encapsulation using polyethylene casing, boxes, and containers); 
- the time required for items to be kept under conditions preventing moisture condensation before their transportation or transfer to unheated premises (sheds etc.) should be determined from Table 3;

- items, machinery, and articles preserved with the VNKh-LF-408 inhibitor can be transported by all kinds of vehicles, vessels, or aircraft. Every stored and transported article should be protected with secondary package preventing mechanical damage and direct exposure to moisture (rain, snow, or fog) (for various internal package items, see protection variant VZ-15 in GOST 9.014).

Electrostatic application of the inhibitor to the metal surface (or into the inside) of a part to be protected is a novel method that should be clarified in more detail; it will be discussed elsewhere.

The developed methodology of application of the VNKh-L-408 inhibitor was used to draw up "The manual of the use of VNKh-L-408 volatile inhibitor in the preservation of parts for protection from atmospheric and biological corrosion". This inhibitor is currently produced at the All-Russia Research Institute of Petrochemical Processes and is employed by a number of enterprises of instrument-making and jewelry industries.

\section{Conclusions}

1. We proposed criteria for inhibitor selection based on the metal corrosion inhibition factors in an environmental chamber.

2. Corrosion tests in an environmental chamber showed that a wide range of ferrous and nonferrous metals are efficiently protected by 1(morpholino)(phenyl)methylbenzotriazole (the VNKh-L-408 inhibitor) in powdered (including electrostatically applied powder) and shaped forms ("tablin" and "granlin").

3. We developed and mastered a technology for pilot scale production of the VNKhL-408 inhibitor.

4. We proposed the main ways for application of the VNKh-L-408 inhibitor for preservation of parts made of ferrous and nonferrous metals, assessed their efficiency, and drew up "The manual of the use of VNKh-L-408 volatile inhibitor in the preservation of parts for protection from atmospheric and biological corrosion".

\section{References}

1. A. I. Altsybeeva, V. V. Burlov, N. S. Fedorova, T. M. Kuzinova and G. F. Palatik, Korroz.: mater., zashch., 2009, 9, 22 (in Russian).

2. Unified System of Protection from Corrosion and Ageing. Means of Temporary Protection from Corrosion. Methods for Determination of the Protective Properties. Introduction, 1991-01-01, GOST (USSR Standard) 9.509, Moscow, Russian State Standard: Standards Publishing House, 1990 (in Russian). 
3. Unified System of Protection from Corrosion and Ageing. Temporary Protection of Articles from Corrosion. General Requirements. - Introduction, 1980-01-01, GOST (USSR Standard) 9.014-78, Moscow, Russian State Standard: Standartinform, 2005 (in Russian).

4. RF Patent 2083719, 1997; Byull. Izobret., 1997, 19, 2 (in Russian).

5. B. Bachman and L. Heisey, J. Am. Chem. Soc., 1946, 68, 12, 2196.

6. K. Nakanishi, Infrared Absorption Spectroscopy, Tokyo, 1962.

7. L. L. Bellamy, The Infra-Red Spectra of Molecules, London, Methuen; New York, Wiley, 1955.

8. P. A. Vinogradov, Konservatsiya izdelii mashinostroeniya (Preservation of MachineBuilding Products), Leningrad, Mashinostroenie, 1986 (in Russian).

9. A. I. Altsybeeva and E. M. Agres, Zh. Prikl. Khim., 1993, 66, 10, 2217 (in Russian). 